\title{
WHEN IS THE COMPOSITION OF TWO POWER SERIES EVEN?
}

\author{
ALAN L. HORWITZ and LEE A. RUBEL
}

(Received 7 February 1992; revised 1 September 1992)

\author{
Communicated by P. C. Fenton
}

\begin{abstract}
If $h$ is the composition of two formal power series $f$ and $g$, and if $h$ is even, what can be said about $f$ and $g$ ? Some partial answers are given here.
\end{abstract}

1991 Mathematics subject classification (Amer. Math. Soc.): 30 B 10.

\section{Introduction}

The work for this paper started with the question - if $f$ and $g$ are entire functions whose composition $f \circ g$ is even, must $f$ or $g$ be even? There are many counterexamples, probably the simplest being the polynomials $f=(z-1)^{2}$ and $g=z+1$. The problem becomes more interesting if one assumes that $g(0)=0$. Then if $f$ and $g$ are polynomials with $f \circ g$ even, $f$ or $g$ must be even (see Theorem 1). However, for entire functions instead of polynomials, there is still, a counterexample, as we describe later. We also give an entire function $f$, with $f(0) \neq 0$, with $f \circ f$ even but $f$ not even - see the Remark following the proof of Theorem 3. However, this is not possible for polynomials (see Theorem 2). Finally, in this vein, we give some results about even compositions of formal power series, from which it follows that there is no formal power series in $g(z)=z^{3}+z^{2}$ which is even (see Proposition 2). The same holds for

(c) 1994 Australian Mathematical Society 0263-6115/94 \$A2.00+0.00

The research of the second author was partially supported by a grant from the National Security Agency. 
$g(z)=z^{8}+z^{5}$, say (see Proposition 3). However, there is a formal power series in $g(z)=z^{4}+z$ which is even. We conclude the paper with the observation that if $f(z)=\sin \pi \sqrt{1-z^{2}}$, then every iterate $f^{[n]}$ of $f$, for $n \geq 2$, is entire, even though $f$ has radius of convergence 1 .

We thank Bruce Reznick for simplifying our proof of Theorem 3.

RESULTS. First we prove some results about when the composition of two polynomials is even.

PROPOSITION 1. Suppose $p$ and $q$ are polynomials, with $p$ nonconstant, $q(0)=0$, and $q$ neither even nor odd. Then $p \circ q$ is neither even nor odd.

PROOF. Assume without loss of generality that $p$ is monic; that is the coefficient of the highest power of $z$ in $p$ is 1 . Let $n=\operatorname{deg} p, m=\operatorname{deg} q$, and write $p(z)=\left(z-\alpha_{1}\right)\left(z-\alpha_{2}\right) \ldots\left(z-\alpha_{n}\right)$. Write

$$
p \circ q(z)=\left(q(z)-\alpha_{1}\right)\left(q(z)-\alpha_{2}\right) \ldots\left(q(z)-\alpha_{n}\right) .
$$

Case 1. $m$ is even.

Since $q$ is not even, we can write $q(z)=a_{m} z^{m}+\cdots+a_{m_{1}} z^{m_{1}}+\cdots$, with $a_{m_{1}} \neq 0$, where $m_{1}$ is the highest odd exponent occurring in the expansion of $q$ about 0 . Now by (1), the coefficient of $z^{m(n-1)+m_{1}}$ in the expansion of $p \circ q$ about 0 must be $n a_{m}^{n-1} a_{m_{1}}$. This is because each factor in (1) except one contributes a factor of $a_{m} z^{m}$, and the remaining factor contributes a factor $a_{m_{1}} z^{m_{1}}$ to $z^{m(n-1)+m_{1}}$, and this happens in $n$ ways. But $m(n-1)+m_{1}$ is odd since $m$ is even and $m_{1}$ is odd. This exponent is the highest odd exponent appearing in (1) because all the integers between $m$ and $m_{1}$ are even. Thus $p \circ q$ cannot be even.

Case 2. $m$ is odd.

Since $\mathrm{q}$ is not odd, we can write $q(z)=a_{m} z^{m}+\cdots+a_{m_{2}} z^{m_{2}}+\cdots$, where $m_{2}$ is the highest even exponent occurring. Note that since $g(0)=0, m_{2}$ must be nonzero, and hence for each $j, q(z)-\alpha_{j}$ contains the nontrivial even term $a_{m_{2}} z^{m_{2}}$. Using (1) as before, we see that the coefficient of $z^{m(n-1)+m_{2}}$ in the expansion of $p \circ q$ about 0 is $n a_{m}^{n-1} a_{m_{2}}$, which is not zero. Now if $p \circ q$ is even, then $n$ must be even since $m$ is odd and $\operatorname{deg}(p \circ q)=m n$. But then $m(n-1)+m_{2}$ is odd, which contradicts $p \circ q$ being even. Again $m(n-1)+m_{2}$ is the highest odd exponent occurring in (1).

The proof that $p \circ q$ is not odd is similar.

REMARK. We only needed the assumption $q(0)=0$ in Proposition 1 in the case where $\operatorname{deg} q$ is odd. 
THEOREM 1. Suppose $p$ and $q$ are polynomials, with $q(0)=0$, and $p \circ q$ even. Then either $p$ or $q$ must be even.

PROOF.

Case 1. $q$ is odd.

Then $p(q(-z))=p(-q(z))$ since $q$ is odd, while $p(q(-z))=p(q(z))$ since $p \circ q$ is even. Hence $p(-q(z))=p(q(z))$ for all $z$, and it follows that $p$ is even since the range of $q$ is the whole complex plane.

Case 2. $q$ is not odd.

If $p$ is constant, we are done. So suppose $p$ is not constant. Then by Proposition $1, p \circ q$ is not even. Hence $q$ must be even.

Our next theorem concerns the compositions of a polynomial with itself. We define the $n$-th iterate $f^{[n]}$ of a formal power series by $f^{[1]}=f, f^{[n+1]}=f \circ f^{[n]}$.

THEOREM 2. Suppose $p$ is a polynomial whose $n$-th iterate $p^{[n]}$ is even for some positive integer $n$. Then $p$ must be even.

(Note that we do not assume that $p(0)=0$.)

PROOF. We treat the case $n=2$. The general case goes the same way. Clearly, $\operatorname{deg} p$ must be even. Now if $p$ is constant, we are done, so assume that $p$ is not constant. Clearly $p$ cannot be odd. If $p$ is not even either, then by Proposition 1 (with $p=q$ ), $p \circ p$ cannot be even. (Note that by Remark (2) following the proof of Proposition 1, we do not need the assumption $p(0)=0$, since deg $p$ is even.) Hence $p$ must be even.

It is interesting to ask whether Theorems 1 and 2 can be extended to entire functions in general. Theorem 1 does not extend - let $f(z)=\cos \pi \sqrt{z+1}$ and let $g(z)=z^{2}+2 z$. Then $f$ and $g$ are entire, $g(0)=0$, and $f \circ g$ is even, but neither $f$ nor $g$ is even. However, the following is open.

QUESTION. Suppose that $f$ and $g$ are entire functions of order less than $1 / 2, g(0)=0$, and that $f \circ g$ is even. Must then either $f$ or $g$ be even?

Theorem 2 does extend if we assume that $f(0)=0$.

THEOREM 3. Suppose that $f$ is a formal power series with vanishing constant term, and that $f^{[n]}$ is even for some positive integer $n$. Then $f$ must be even. 
PROOF. Clearly, $f$ cannot be odd (unless $f=0$ ). Now if $f$ is not even, then by the theorem proved in [2], $f^{[m n]}(z)=z$ for some positive integer $m$. Thus $\left(f^{[n]}\right)^{[m]}=z$, which is impossible if $f^{[n]}$ is even.

REMARK. Theorem 3 does not hold in general if $f(0) \neq 0$. For example, let $f(z)=1-\sin \frac{\pi}{2} z$. Then $f \circ f(z)=1-\cos \left[\frac{\pi}{2} \sin \frac{\pi}{2} z\right]$, which is even, while $f$ is clearly not even.

We now extend Proposition 1 and Theorem 1 in certain cases to formal power series. Write

$$
\begin{array}{ll}
f(z)=\sum_{k=N}^{\infty} a_{k} z^{k}, & a_{N} \neq 0 . \\
g(z)=\sum_{k=M}^{\infty} b_{k} z^{k}, & b_{M} \neq 0 .
\end{array}
$$

First, we give conditions on $g$ which imply that $f \circ g$ cannot be even.

PROPOSITION 2. Let $f$ and $g$ be formal power series given by (2) and (3), and suppose $g$ is not even. Assume also that $M$ is even. Then $f \circ g$ cannot be even.

PROOF. Write

$$
\begin{aligned}
f \circ g(z)= & a_{n}\left(b_{M} z^{M}+\cdots+b_{n} z^{n}+\sum_{k=n+1}^{\infty} b_{k} z^{k}\right)^{N} \\
& +a_{N+1}\left(b_{M} z^{M}+\cdots+b_{n} z^{n}+\sum_{k=n+1}^{\infty} b_{k} z^{k}\right)^{N+1}+\cdots
\end{aligned}
$$

where $n$ is the smallest odd integer such that $b_{n} \neq 0$. Then the smallest odd exponent in the first term in the right-hand side of (4) is clearly $M(N-1)+n$ since all the exponents between $M$ and $n$ are even. Also, any odd exponent occurring in any other term on the right-hand side of (4) must have exponent at least $M N+n>M(N-1)+n$. Since $a_{N} \neq 0, f \circ g$ cannot be even.

THEOREM 4. Suppose $f$ and $g$ are formal power series given by (2) and (3) with $f \circ g$ even. Suppose that $M$ is even. Then either $f$ or $g$ must be even.

PROOF. Suppose that $f$ is not even. Since $M$ is even, $g$ is not odd. If $g$ were not even either, then $f \circ g$ would not be even, by Proposition 2. Hence $g$ must be even. 
PROPOSITION 3. Let $f$ and $g$ be formal power series given by (2) and (3), where $M$ is odd and $g$ is not odd. Let $n$ be the smallest even integer such that $b_{n} \neq 0$. If $M>n / 2$, then $f \circ g$ cannot be even.

PROOF. We consider two cases.

Case 1. $\quad N$ is odd. Then $M N$ is odd, and using (4) again, we see that $M N$ is the lowest exponent occurring in $f \circ g$. Since $a_{N} \neq 0, f \circ g$ is not even.

Case 2. $\quad N$ is even.

Then the lowest odd exponent occurring in the first sum in (4) is $M(N-$ $1)+n$, since any exponents of $g$ between $M$ and $n$ are odd. The only other way to get $M(N-1)+n$ as an exponent in (4) is by terms of the form $b_{m_{1}} \ldots b_{m_{N+r}} z^{m_{1}+\cdots+m_{N+r}}$, where $m_{j} \geq M$ for $j=1, \ldots, N+r$ and $r \geq 1$. Now let $m_{1}+\cdots+m_{N+r}=M(N+r)+p_{1}+\cdots+p_{N+r}$, where $p_{j}=m_{j}-M$. If $m_{1}+\cdots+m_{N+r}=M(N-1)+n$, then $n=(r+1) M+p_{1}+\cdots+p_{N+r} \geq$ $(r+1) M \geq 2 M$, which contradicts the assumption that $n<2 M$. Hence, there is no cancellation of the odd power with exponent $M(N-1)+n$ in the first term on the right-hand side of (4), so that $f \circ g$ cannot be even.

REMARK. Theorem 4 does not hold in general without the restriction $n<2 M$. For example, as noted earlier, take $f(z)=\cos 2 \pi \sqrt{z+1}$ and $g(z)=z^{2}+2 z$. Or take $g(z)=z^{4}+z$, say, and let $f(z)=z^{2} \circ g^{-1}(z)$.

One could also put restrictions on $f$. For example, we prove:

THEOREM 5. Let $f$ and $g$ be formal power series given by (2) and (3), and suppose that $g$ is neither even nor odd. If $N$ is odd, then $f \circ g$ cannot be even.

PROOF. If $M$ is even, then Theorem 5 follows from Proposition 2, whether $N$ is odd or not. If $M$ is odd, then the smallest exponent occurring in (4) is $M N$, which is odd.

We conclude with an example that is only loosely related to the rest of this paper.

EXAMPLE. Let $f(z)=\sin \pi \sqrt{1-z^{2}}$. Then $f$ has a finite radius of convergence, but every iterate $f^{[n]}$, for $n \geq 2$, has infinite radius of convergence. Just observe that

$$
f^{[2]}(z)=\sin \left[\pi \cos \pi \sqrt{1-z^{2}}\right], \quad f^{[3]}(z)=\sin \pi\left[\cos \pi\left(\cos \pi \sqrt{1-z^{2}}\right)\right],
$$

etc. 
REMARK. As we noted earlier, there is a non-constant function $f$, analytic at 0 , such that $f\left(z^{4}+z\right)$ is even. But there is no such entire $f$. For suppose $f$ were entire and non-constant, with $f\left(z^{4}+z\right)$ even. Then $f(p(z))=f(q(z))$, where $p(z)=z^{4}+z$ and $q(z)=z^{4}-z$. By the theorem in [1], either $p(z)=\lambda q(z)+\beta$ for some constants $\lambda$ and $\beta$ or $p(z)=(r(z))^{2}+k$ for some entire function $r$ and some constant $k$. It is easy to verify that neither of these can happen.

It is interesting (see [3]) that if $(f \circ g)(v z)=(f \circ g)(z)$, where $v$ is an $m$-th root of unity, $m \geq 3$ and prime, then $g(v z)=g(z)$, where $f$ is a transcendental entire function and $g$ is a polynormal of degree $n$, and $m \mid n$. Note that this does not hold if $m=2$ and $v=-1$, as the example $f(z)=\cos 2 \pi \sqrt{z+1}$, $g(z)=z^{2}+2 z$ shows.

\section{References}

[1] I. N. Baker and Fred Gross, 'On factorizing entire functions', Proc. London Math. Soc 17 (1968), 69-76.

[2] Bruce Reznick, 'When is the iterate of a formal series odd?', J. Austral.Math. Soc. (Series A) 28 (1979), 62-66.

[3] Xin-Hou-Hua, 'Uniquely factorizable entire functions', J. Math. Anal. Appl. 153 (1990), 11-17.

Pennsylvania State University

25 Yearsley Mill Road

Media, PA 19063

U.S.A
University of Illinois

1409 West Green Street

Urbana, IL 61801

U.S.A 